\title{
AVALIAÇÃO NUTRICIONAL DE ESTUDANTES UNIVERSITÁRIOS DO SEXO FEMININO
}

\author{
Elizabeth Fujimori* \\ Ida Maria Vianna de Oliveira** \\ Mariângela Abate de Lara Soares*** \\ Ana Cristina Osso***
}

FUJIMORI, E. et al Avaliaçāo nutricional de estudantes universitários do sexo feminino. Rev. Esc. Enf. USP, v.28, n.1, p. 72-82, abril, 1994.

A auto-avaliaçào do estado nutricional, utilizada como estratégia de ensino no Curso de Graduaçào em Enfermagem, subsidiou o presente trabalho. Com esse fim, as 50 alunas do $3^{9}$ semestre (idade média de 21,7 anos) registraram seu consumo de alimentos por trés dias consecutivos e suas medidas de peso e altura $A$ análise da adequaçào do consumo mostrou que $98 \%$ das alunas apresentaram ingestảo insuficiente (abaixo de 90\% do recomendado pela National Research Council - 1980) em pelo menos um dos nutrientes analisados (proteina, cálcio, ferro e vitaminas A, B1, B2, C e niacina). Constatou-se ingestāo inadequada de cálcio em $80 \%$ das alunas, de vitamina $A$ em 72\%, de ferro em 64\%, de vitamina B1 e B2 em 74 e 56\% respectivamente e de vitamina C em 34\%. A freqüência e a gravidade da inadequaçāo foi maior para cálcio, vitamina $A$ e ferro: 42,20 e 16\% das alunas consumiram menos que $60 \%$ da recomendaçào, evidenciando uma dieta deficiente em vitaminas e minerais.

UNITERMOS: Estado nutricional. Estudo dietético. Consumo alimentar. Estudantes universitários.

\section{INTRODUÇĀO}

Tradicionalmente, no Brasil, os estudantes universitários não têm sido considerados como um grupo vulnerável às deficiências nutricionais. Isto se justifica, em parte, pela faixa etária - adolescentes e jovens - que comparada a outros segmentos populacionais, apresenta baixo risco de mortalidade e suscetibilidade a doenças ${ }^{21}$. Adicionalmente, tem se vinculado o acesso ao

Assistente do Departamento de Enfermagem em Saúde Coletiva da Escola de enfermagem da Universidade de Sáo Paulo.

* Profensor Doutor do Departanento de Enfermagem em Saúde Coletiva da Escola de Enfermagem da Universidade de Sáo Paulo.

*** Ex-alunas - monitoras da Disciplina Nutriçáo Aplicada i Enfermagem do Departamento de Enfermagem em Saúde Coletiva da Escola de Enfermagem da Universidade de São Paulo. 
ensino superior às classes de maior poder aquisitivo e estas a uma maior disponibilidade e consumo de alimentos ${ }^{1,17,18}$.

Entretanto, inúmeros trabalhos têm evidenciado carências nutricionais em universitários 3,7,8,9,10,11,12,14,16,20 de diferentes regiōes e países. Esses estudos revelam que, embora o consumo médio de nutrientes seja geralmente adequado (exceto ingestão de ferro em mulheres) é significativa a percentagem de estudantes, cujo consumo individual se mostra inferior aos níveis recomendados.

Em estudo anterior, também realizado com estudantes de enfermagem do sexo feminino, FUJIMORI et al ${ }^{7}$ encontraram um número significativo de estudantes com consumo deficiente sobretudo em ferro, vitamina A, tiamina e riboflavina; apesar do consumo médio se apresentar adequado.

Para JACOBOVITS et al ${ }^{12}$ e HERNON et al ${ }^{9}$, a inadequaçāo nutricional observada em mulheres universitárias norte-americanas decorre da ingestão insuficiente dos diferentes grupos de alimentos, associada a um consumo calórico menor que $1.200 \mathrm{cal}^{9}$.

Alteraçōes no padrāo alimentar, refletindo mudanças no estilo de vida e maior envolvimento com atividades acadêmicas ${ }^{8,9,15}$, associadas ou não a consumo de dietas hipocalóricas visando perda de peso 10.11 .15 e rejeiçāo de determinados grupos de alimentos ${ }^{12}$ são outros fatores que têm sido identificados como determinantes do inadequado consumo de alimentos e nutrientes por estudantes universitários.

Considerando a existência de poucos estudos sobre o assunto no Brasil e a oportunidade de, trabalhando com alunas do curso de graduação em Enfermagem, obter dados de auto-a valiaçāo nutricional, realizou-se o presente trabalho. Seu objetivo foi avaliar nảo só o padrào alimentar, mas também a adequação do peso (em relação à altura) e do consumo de nutrientes destas estudantes.

\section{METODOLOGIA}

POPULAÇĀO - O grupo de estudo foi constituido por 50 estudantes do sexo feminino, alunas do $3^{2}$ semestre do Curso de Graduação em Enfermagem da Escola de Enfermagem da Universidade de São Paulo, no ano de 1988.

AVALLAÇĀO ANTROPOMÉTRICA - Verificou-se a estatura e o peso corporal de cada estudante utilizando-se balança antropométrica Filizola, (com capacidade para $150 \mathrm{~kg}$ e escala de $100 \mathrm{~g}$ ). As medidas foram tomadas durante o período de inquérito alimentar, sem calçados e com roupa leve, e em horário anterior à refeição (almoço). O peso ideal foi determinado através do método proposto por WEST ${ }^{22}$ utilizando a relação: $\mathrm{PI}=$ altura $(\mathrm{m})^{2} \times$ fator (f), sendo $f=20,9$ (valor para mulheres sem sapatos e com roupa leve). Para classificação do estado nutricional, foram utilizados as seguintes faixas de adequação: baixo peso (Z\%), normal (90|-110\%), sobrepeso $(110 \mid-120 \%)$ e obesidade $(/ 120 \%)^{13.16}$. 
AVALIAÇẢO DO CONSUMO ALIMENTAR - Foi feita através de registro individual do consumo de alimentos por três dias consecutivos (nào incluindo o fim de semana). Os dados foram coletados considerando porçóes e medidas caseiras posteriormente convertidas em gramas ${ }^{4}$. $O$ teor de nutrientes nos alimentos foi estimado com base na Tabela de Composição de Alimentos da Fundaçào Instituto Brasileiro de Geografia e Estatística (FIBGE) ${ }^{5}$. A adequação nutricional foi calculada para energia, proteína, cálcio, ferro, vitamina A, tiamina, riboflavina, niacina e vitamina C, comparando-se os nutrientes contidos nos alimentos consumidos (média per capita/dia) com as necessidades nutricionais. Para o cálculo das necessidades de nutrientes utilizou-se as recomendaçōes da National Research Council (NRC) - USA ${ }^{6}$ para sexo e faixa etária correspondente, sendo que para energia e proteína considerou-se, adicionalmente, o peso corporal de cada estudante. Para cada nutriente, o consumo foi considerado inadequado quando inferior a $90 \%$ da recomendação.

Calculou-se também a distribuição calórica da dieta, ou seja, a contribuição de cada nutriente energético (glícides, lípides e proteínas) no total de calorias consumidas, além da percentagem de proteínas e lipídios de origem animal em relação ao total desses nutrientes na dieta.

CONSUMO DOS DIFERENTES GRUPOS DE ALIMENTOS - Foi estimada a quantidade média consumida dos alimentos correspondentes aos diferentes grupos da dieta normal (leite e derivados; carne, ovos e leguminosas; hortaliças; frutas; cereais e feculentos; açúcares, doces e gorduras) e comparada com a porção mínima diária recomendada para estes grupos ${ }^{13,16}$.

Também foram levantados 08 antecedentes familiares relativos à incidência de doenças crônico-degenerativas, associadas direta ou indiretamente à nutrição (diabetes, hipertensão, doenças cardiovasculares).

\section{RESULTADOS E DISCUSSÃO}

AVALIAÇĀO ANTROPOMÉTRICA - A Tabela 1 apresenta as médias de idade, estatura e peso corporal do grupo estudado. Observa-se que apesar da variaçāo encontrada na idade ( 19 a 27 anos), a média se aproxima do valor mínimo $(21,7+-2,14$ anos $)$, uma vez que $74 \%$ das estudantes que participaram do estudo tinham de 19 a 22 anos, e $37 \%$ do total tinham menos de 20 anos, sendo consideradas adolescentes segundo a OMS ${ }^{19}$.

O peso corporal médio das alunas $(55,6 \mathrm{~kg}+-9,15)$, excede em cerca de $2 \mathrm{~kg}$ o peso ideal médio calculado para a altura $(53,6 \mathrm{~kg}+-4,92)$ e se assemelha a valores encontrados por HERNON et al ${ }^{9}$ em estudantes americanas do sexo feminino de mesma faixa etária. 
Tabela 1 - Valores médios, minimos e máximos da idade, altura e peso corporal do grupo estudido.

\begin{tabular}{lrrr}
\hline Parámetros & Média & \multicolumn{2}{c}{ Valor } \\
& & Mínimo & Máximo \\
\hline Idade, anos & $21,7+-2,14$ & 19,0 & $\mathbf{2 7 , 0}$ \\
Altura, cm & $160,0+-7,36$ & 144,0 & 184,0 \\
Peso atual (PA), kg & $55,6+-9,15$ & 42,7 & 83,4 \\
Peso ideal (PI), kg & $53,6+-4,92$ & 43,3 & 62,6 \\
\hline
\end{tabular}

Esta observação é reforçada pelos resultados apresentados na Tabela 2 que mostram $24 \%$ das alunas com excesso de peso (sobrepeso e obesidade) em relação a $64 \%$ com peso adequado. A percentagem de alunas que apresentaram baixo peso (12\%) foi bem menor que a observada em estudo anterior (21\%), também realizado com estudantes de enfermagem 7 , enquanto a freqüência de excesso de peso foi maior ( $24 \%$ e $17 \%$ respectivamente). Verifica-se que no periodo compreendido entre os dois trabalhos (aproximadamente 6 anos) houve uma redução de $43 \%$ no número de estudantes com baixo peso e um aumento proporcional (41\%) dos casos de excesso de peso.

Os dados da Pesquisa Nacional sobre Saúde e Nutrição (PNSN) evidenciam essa mesma tendência para mulheres brasileiras mostrando, entretanto, um aumento mais acentuado (49\%) da prevalência de excesso de peso num período de 15 anos (1974-1989) ${ }^{2}$.

Tabola 2 - Distribuiçào do grupo estudado segundo a adequaçio do peso corporal.

\begin{tabular}{lrr}
\hline Adequaçio do peso corporal $^{1}$ & $\mathbf{n}^{\circ}$ & $\%$ \\
\hline Baixo peso ( 90\%) & 6 & 12,0 \\
Normal (90 |- 110\%) & 32 & 64,0 \\
Sobrepeso (110 |- 120\%) & 5 & 10,0 \\
Obeeidade ( 120\%) & 7 & 14,0 \\
\hline TOTAL & 50 & 100,0 \\
\hline
\end{tabular}

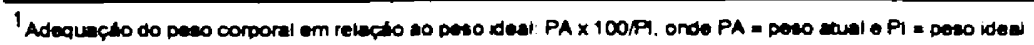

AVALIAÇÃO DO CONSUMO ALIMENTAR - O consumo médio de energia e de nutrientes das estudantes é apresentado na Tabela 3 , a percentagem média de adequaçāo na Figura 1 e algumas relaçōes entre os nutrientes na Tabela 4. 
Tabela 3 . Valores médios de energra e autrientes consumidos pelo grupo estudado.

Nutrientes

Ingestáo

Recomendada ${ }^{1} \quad \operatorname{Media}^{2}$ (per capita/dia)

\begin{tabular}{lrr}
\hline Energia, Cal & $2085,3+346,01$ & $2126,6+493,90$ \\
Proteings, g & 46,0 & $76,2+18,17$ \\
Cálcio, mg & 800,0 & $529,1+262,55$ \\
Ferro, mg & 18,0 & $14,2+3,76$ \\
Vit. A. gRE & 800,0 & $659,2+295,57$ \\
Tiamina, mg & 1,1 & $0,9+0,23$ \\
Riborlavina, mg & 1,3 & $1,1+0,29$ \\
Niacina.EN & 14,0 & $17,6+4,80$ \\
Vit. C, mg & 60,0 & $\mathbf{8 8 , 7}+\mathbf{4 7 , 1 0}$
\end{tabular}

1 - RDA $1980(6)$

2 - módia + deovio padrâo

$3-\mathrm{gRE}=\mathrm{g}$ de Rotinol Equivalente

4- EN = Equivalente de Niacina

\section{FIGURA 1}

Adequeçāo média do consumo, em percentagem do grupo estudado

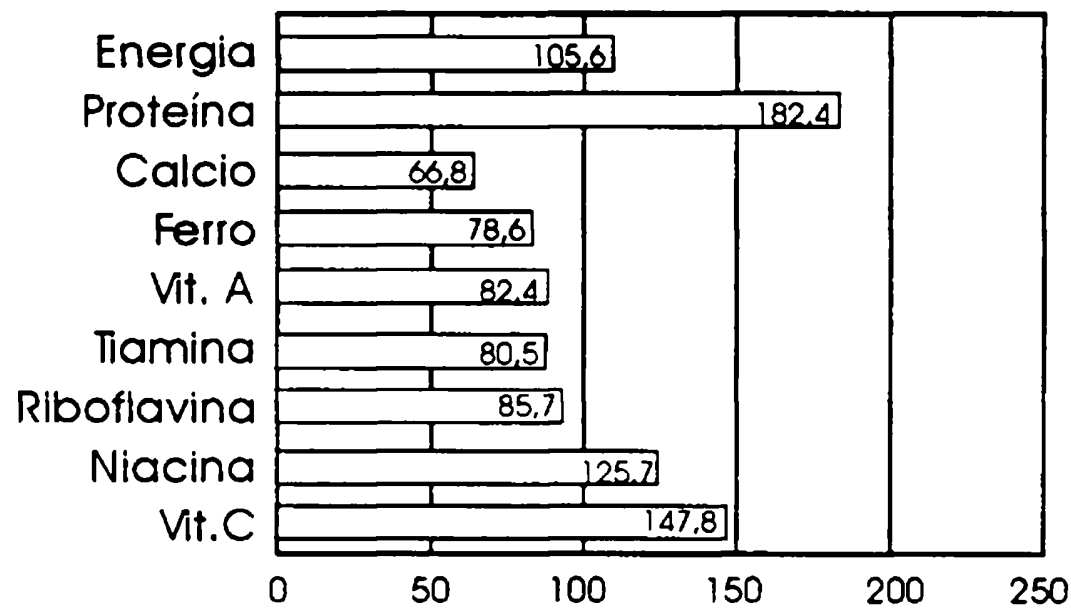

Observa-se uma ingestão média acima da recomendada pelo NRC - 1980 ${ }^{6}$ para energia, proteína, niacina e vitamina C. Para os demais nutrientes (cálcio, ferro, vitamina A, tiamina e riboflavina), o consumo médio apresentou-se inadequado, variando de $14 \%$ (riboflavina) a $33 \%$ (cálcio) abaixo da recomendação. 
Esses resultados são semelhantes àqueles obtidos por ISHIGAKI; SU. ZUKI ${ }^{11}$ e MAEKAWA et al ${ }^{14}$ que encontraram baixo consumo de cálcio, ferro e vitamina A em estudantes universitárias japonesas. DRISKELL et al ${ }^{3}$, da mesma forma, verificaram inadequação de ferro e tiamina, mas não estuda. ram vitamina A e cálcio. HERNON et al ${ }^{9} \mathrm{e}$ JAKOBOVITS et al ${ }^{12}$, no entanto, verificaram entre jovens americanas consumo maior de vitamina $A$, vitamina $\mathrm{C}$, tiamina, riboflavina e cálcio em relaçāo ao obtido neste estudo, mas menor de ferro e energia.

Tabela 4 - Distribuiçio calórica da dieta e outras relaçóes entre os nutrientes

\begin{tabular}{|c|c|c|}
\hline Rolaçio dos nutrientes & $\%$ do total consumido & Recomendaçao ${ }^{1}(9)$ \\
\hline Energéticos proteicas/VCT ${ }^{2}$ & $15,5+\cdot 8,3$ & $12 \cdot 15$ \\
\hline Glicides/VCT & $50,5+\cdot 9,3$ & 50.55 \\
\hline Lipides/VCT & $34,3+-6,4$ & $30-35$ \\
\hline $\begin{array}{l}\text { Proteina de origem } \\
\text { animal/total de proteinas }\end{array}$ & $59,0+-14,7$ & 33,3 \\
\hline $\begin{array}{l}\text { Lípides saturados/total de } \\
\text { lípideo }\end{array}$ & $35,0+-18,4$ & \\
\hline Ferro animal/Total de Ferro & $33,5+-12,5$ & - \\
\hline
\end{tabular}

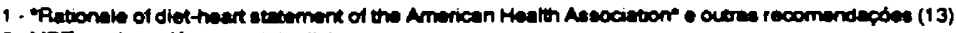

2 - VCT = vaior calorico total da dints

A contribuição de cada nutriente energético no total das calorias consumidas foi similar àquela observada por diversos autores $7,8,9,20$, mas ligeiramente superior no que toca ao percentual calórico proveniente dos glícides (Tabela 4). Pode-se considerar que a distribuiçāo calórica da dieta do grupo estudado encontra-se dentro dos níveis recomendados, para calorias protéicas e glicídicas. Entretanto, $\mathbf{4 2 \%}$ das alunas apresentaram consumo das calorias, provenientes de lípides, maior do que $35 \%$, excedendo portanto o limite máximo recomendado pelo American Hearth Association ${ }^{13}$. HERTZLER; FRARY ${ }^{10}$ também verificaram consumo de dieta rica em gordura em estudantes universitários. Esse fato merece atenção, uma vez que a ingestāo elevada de lípides tem sido associada a maior incidência de problemas cardiovasculares e $64 \%$ das estudantes referiram hipertensăo e/ou outras enfermidades cardiovasculares na família.

A ingestão média de proteína pelo grupo estudado excedeu não só a recomendação de $0,8 \mathrm{~g} / \mathrm{kg}$ de peso corporal por dia (Figura 1), como também a de que, pelo menos um terço da proteína dietética seja de origem animal (Tabela 4) ${ }^{16}$. O consumo de proteína de origem animal encontrado em estudo anterior ${ }^{7}$ foi mais elevado, atingindo $63 \%$ ou seja quase dois terços do total. Já MAEKAWA et al ${ }^{14}$ verificaram um consumo da ordem de $55 \%$, em dieta de estudantes universitárias japonesas. 
Tabela 5 - Distribuição do grupo estudado segundo a adequaçáo do consumo de energia e nutrientes.

Alunas

\begin{tabular}{|c|c|c|c|c|}
\hline \multirow[t]{2}{*}{ Nutrientes } & \multicolumn{2}{|c|}{ Ingestão adequada } & \multicolumn{2}{|c|}{ Ingestao inadequada } \\
\hline & $\mathrm{N}^{2}$ & $q$ & $\mathrm{~N}^{\mathrm{O}}$ & $\%$ \\
\hline Energia & 32 & 64 & 18 & 36 \\
\hline Proteina & 48 & 96 & 02 & 04 \\
\hline Cálcio & 10 & 20 & 40 & 80 \\
\hline Ferro & 18 & 36 & 32 & 64 \\
\hline Vitamina A & 14 & 28 & 36 & 72 \\
\hline Tiamina & 13 & 26 & 37 & 74 \\
\hline Riboflavina & 22 & 44 & 28 & 56 \\
\hline Niacina & 43 & 86 & 07 & 14 \\
\hline Vitamina C & 33 & 66 & 17 & 34 \\
\hline
\end{tabular}

1. Consumo menter a soto de recomendacto

A Tabela 5 demonstra que apesar do consumo médio de energia, proteína, niacina e vitamina $C$ se apresentar adequado (Figura 1 e Tabela 3) pelo menos $30 \%$ do grupo estudado nāo consumiu a quantidade recomendada de energia e vitamina C, $14 \%$ não consumiu a de niacina e $4 \%$ a de proteína.

Numa análise geral dos resultados de consumo verifica-se que nenhum dos nutrientes estudados foi consumido em quantidade adequada por todas as estudantes (Tabela 5). Adicionalmente, $98 \%$ das alunas apresentam ingestão insuficientes em pelo menos um deles, ou seja, do total das 50 alunas apenas uma mostrou ingestāo adequada de todos os nutrientes.

Observa-se, ainda, pela Tabela 5 a alta percentagem de estudantes que apresentaram ingestāo insuficiente de cálcio, ferro, vitaminas $A$, tiamina e riboflavina.

Ao se avaliar o grau dessa insuficiência (Tabela 6), verifica-se que o cálcio foi o nutriente que apresentou maior gravidade de inadequação, pois $80 \%$ das alunas apresentaram ingestāo inadequada, e $42 \%$ não chegaram a consumir $60 \%$ da recomendaçảo. Isso mostra uma inadequaçāo em cálcio muito maior na dieta dessas alunas do que aquela encontrada em dieta de estudantes americanas $10,12,20$. Inversamente, em relaçảo ao ferro, a inadequação apresentou-se menor e menos grave. Mesmo assim, observa-se pela Figura 1 que o ferro é o nutriente mais deficiente depois do cálcio. Aproximadamente dois terços (64\%) das alunas apresentaram consumo inadequado do mineral (Tabela 5), e $16 \%$ consumiram menos que $60 \%$ do recomendado (Tabela 6). Além disso, apenas um terço do ferro ingerido foi de origem animal (Tabela 4), o que sugere maior gravidade de inadequação, uma vez que o ferro de origem vegetal, de menor biodisponibilidade, predomina na dieta. 
Tabela 6 - Distribuiçáo do grupo estudado segundo a gravidade da inadequacáo alimentar

\begin{tabular}{|c|c|c|c|c|c|c|c|c|}
\hline \multirow{3}{*}{ Nutrientes } & \multicolumn{8}{|c|}{ Faixas de adequaçảo } \\
\hline & \multicolumn{2}{|c|}{01.30} & \multicolumn{2}{|c|}{$30 \mid-60$} & \multicolumn{2}{|c|}{601.90} & \multicolumn{2}{|c|}{$90 \mathrm{ou}+$} \\
\hline & $\mathrm{N}^{8}$ & $\%$ & $\mathrm{~N}^{2}$ & $q$ & $\mathrm{~N}^{2}$ & $\%$ & $N^{2}$ & $\%$ \\
\hline Energia & 0 & 0 & 5 & 10 & 13 & 26 & 32 & 64 \\
\hline Proteins & 0 & 0 & I & 2 & 1 & 2 & 48 & 96 \\
\hline Cálcio & 4 & 8 & 17 & 34 & 19 & 38 & 10 & 20 \\
\hline Ferro & 1 & 2 & 7 & 14 & 24 & 48 & 18 & 36 \\
\hline Vitamina A & 2 & 4 & 8 & 16 & 26 & 52 & 14 & 28 \\
\hline Tiamina & 1 & 2 & 5 & 10 & 31 & 62 & 13 & 26 \\
\hline Riboflavina & 0 & 0 & 7 & 14 & 21 & 42 & 22 & 44 \\
\hline Niacina & 0 & 0 & 2 & 4 & 5 & 10 & 43 & 86 \\
\hline Vitamina C & 1 & 2 & 3 & 6 & 13 & 26 & 33 & 66 \\
\hline
\end{tabular}

Embora, em estudo já mencionado ?, alunas do Curso de Graduação em Enfermagem não apresentassem ingestão média inadequada de vitamina $\mathrm{A}$, no presente trabalho verificou-se que o consumo médio dessa vitamina atingiu somente $82 \%$ da recomendaçảo (Tabela 3 e Figura 1). Apesar disso, em ambos os estudos cerca de $70 \%$ das alunas apresentaram consumo individual inferior a $90 \%$ de adequação.

Analisando a gravidade da inadequação dessa vitamina observou-se, no presente estudo, que $20 \%$ das estudantes consumiram menos que $60 \%$ do recomendado (Tabela 6). Resultados de OSTRON; LABUZA ${ }^{20}$ mostram uma inadequaçāo, ainda, mais grave: $38 \%$ das jovens americanas que estudaram nảo atingiu esse nível de recomendação. JAKOBOVITS et al ${ }^{12}$ observaram uma menor percentagem $(3,6 \%$ ) de estudantes ingerindo menos que $60 \%$ do recomendado, enquanto HERTZLER; FRARY ${ }^{10}$, mais recentemente, obtiveram 29\% de estudantes universitários com ingestão menor que 70\% da RDA.

Quanto às vitaminas do complexo $B$, uma análise comparativa evidencia inadequação mais grave de tiamina em relação à riboflavina e niacina (Figura 1). Tanto neste estudo, quanto nos americanos já citados ${ }^{12,20}$, a percentagem de jovens com consumo de tiamina inferior a $60 \%$ é de aproximadamente $10 \%$. Para riboflavina, os nossos resultados foram piores que os apresentados por aqueles estudos, enquanto para niacina ocorreu o inverso.

CONSUMO DOS DIFERENTES GRUPOS DE ALIMENTOS - A maior freqüência e gravidade com que se apresentam as deficiências de nutrientes, sobretudo minerais e vitaminas, no grupo estudado pode ser justificada pelo consumo insuficiente de alimentos como leite e derivados e hortaliças (Tabela 6).

Observa-se, que o consumo médio desses grupos de alimentos foi de aproximadamente dois terços do recomendado, sendo que apenas $24 \%$ das estudantes ingeriam quantidades adequadas de leite e derivados e $14 \%$ de hortaliças. Além disso, somente $26 \%$ das alunas consumiram hortaliças de 
cor verde escuro ou amarelo intenso, consideradas fontes de vitamina A. Os demais grupos de alimentos (carnes, ovos e leguminosas, frutas e cereais) nào só foram consumidos em quantidades médias consideradas adequadas, mas uma menor percentagem de alunas tiveram ingestāo abaixo da recomendada, o que provavelmente garantiu a adequação protéico-energética e de vitamina C para a maioria das alunas.

Tabela 7 - Consumo médio dos diferentes grupos de alimentos e adequaçáo em relaçáo à dieta normal recomendada (16).

\begin{tabular}{|c|c|c|c|c|c|c|}
\hline \multirow{3}{*}{$\begin{array}{l}\text { Grupo de } \\
\text { alimentos }\end{array}$} & \multirow{3}{*}{$\begin{array}{l}\text { Consumo } \\
\text { médio (g) }\end{array}$} & \multicolumn{5}{|c|}{ Adequação do consumo } \\
\hline & & \multirow[t]{2}{*}{ MÉDIA } & \multicolumn{2}{|c|}{ INADEQUADO } & \multicolumn{2}{|c|}{ ADEQUADO } \\
\hline & & & $N^{2}$ & \% & $\mathrm{N}^{\circ}$ & $\not$ \\
\hline $\begin{array}{l}\text { Leite } \\
\text { derivados }\end{array}$ & $175,0+-117,5$ & $68,6+-54,4$ & 38 & 76,0 & 12 & 24,0 \\
\hline $\begin{array}{l}\text { Cames, ovos } \\
\text { e leguminosas }\end{array}$ & $212,1+\cdot 91,1$ & 103,2 & $+-37,9$ & 18 & 36,0 & 32 \\
\hline Hortaliças & $127,4+\cdot-108,9$ & $63,4+-54,6$ & 43 & 86,0 & 07 & 14,0 \\
\hline Frutas & $239,8+-145,4$ & $121,1+\cdot 71,0$ & 16 & 32,0 & 34 & 68,0 \\
\hline $\begin{array}{l}\text { Cerea is e } \\
\text { feculentoo }\end{array}$ & $274,3+\cdot 134,3$ & $136,5+-67,8$ & 11 & 22,0 & 39 & 78,0 \\
\hline $\begin{array}{l}\text { Doces, mel, } \\
\text { açúcar }\end{array}$ & $074,9+-047,5$ & - & - & - & - & . \\
\hline Gorduras 1 & $093,9+\cdot 033,2$ & - & . & - & - & . \\
\hline
\end{tabular}

1)6leo. creme de berte, mantiven, maconese molno threro

\section{CONCLUSŌES}

Considerando os resultados obtidos no presente estudo vale ressaltar nāo só a incidência de $24 \%$ de casos de sobrepeso e obesidade como a elevada contribuição dos lípides no total das calorias consumidas pelas estudantes.

Também se evidencia que, embora os valores médios mostrem como adequada a ingestão de calorias, proteína, niacina, e vitamina $\mathrm{C}$, nenhum desses e dos demais nutrientes foram consumidos adequadamente pelo total (100\%) do grupo estudado. Outrossim, $98 \%$ das alunas apresentaram ingestāo insuficiente em pelo menos um dos nutrientes analisados, constatando-se deficiências quantitativas mais freqüentes e mais graves para os nutrientes: cálcio, ferro, vitamina A, tiamina e riboflavina, o que poderia estar refletindo o consumo inadequado principalmente de dois grupos de alimentos - leite e derivados e hortaliças.

Tais observaçōes justificam propostas de continuidade de estudos semelhantes averiguando tanto a correlação do baixo consumo desses alimentos com possíveis alteraçōes no padráo alimentar decorrentes das atividades em período integral do Curso de Graduação em Enfermagem, como o reflexo da auto-avaliaçáo nutricional na reorientação alimentar das estudantes. 
FUJIMORI, E. et al Nutrition evaluation of female college students. Rev. Esc. Enf. USP. v. 28,n.1,p 72.82, Apr.1994.

The self-evaluation of nutritional status, utilized as a teaching strategy of $y$ of introductory nutrition course at Nursing School (Sâo Paulo's University), gave a e a subsidy to this work. With this purpose, 50 female university students 3rd'semester ster (mean age was 21,7) were required to complete a three days food record and report their rt thei appraximate heigth and weigth. The analysis of food intake adequacy indicated that $d$ that 98\% of the students had insufficient intake (less than $90 \%$ of recommended of National tional Research Council - 1980) of, at least, one of the nutrients (protein, calcium, iron, iron, vitamin $A, B 1, B 2, C$ and niacin). It was evidenced an inadequate intake (less than than $90 \%$ of RDA) of calcium ( $80 \%$ of the students), vitamin $A(72 \%)$, iron (64\%), vitamin min $B 1$ (74\%), vitamin $B 2$ (56\%) and vitamin $C$ (34\%). The inadequate intake was more rore frequently and serious (less than 60\% RDA) for calcium (42\%), vitamin A (20\%) and 'and iron (16\%) showing a vitamins and minerals deficient diet.

UNITERMS: Nutrition status. Diet studies. Food intake. College students.

\section{REFERÊNCIAS BIBLIOGRÁFICAS}

01. BATISTA FILHO, M.; BARBOSA, N.P. Alimentação e nutrição no Braşil 1974 1984. Brasília, Ministério da Saúde - Instituto Nacional de Alimentaçảo e Nutriçăo, 1985.

02. COITINHO, D.C. et al Condiçōes nutricionnis da populaçào brasileira: adultos e idosos. Brasília, Ministério da Saúde - Instituto Nacional de Alimentos e Nutriçäo, 1991.

03. DRISKELI, J.A. et al Nutritional status of white college students in Virginis. JAm.Diet_Ansocen v.74, n.1, p. 32-5, 1979.

04. ESCOLA DE ENFERMAGEM DA USP.Departamento de Enfermagem em Saúde Coletiva. Tabela de conversio de medidas caseiras em gramas. Sāo Paulo, 1985/mimeografado/

05. FUNDAÇĀO INSTITUTO BRASILEIRO DE GEOGRAFIA E ESTATÍSTICA (FIBGE) Estudo nacional da despesa familiar - ENDEF: tabelas de composiçáo de alimentos Rio de Janeiro, 1977.

06. FOOD AND NUTRITION BOARD - Recommended dietary allowances, 9th rev.Washington, National Academy of Sciences - National Research Council, 1980.

07. FUJIMORI, E. et al Consumo alimentar de estudantes universitários do sexo feminino. Rov.Esc.Enf.USP, v.20, n.2, p.115-24, 1986.

08. GOTTSCHALK, P.L. et al Nutrient intakes of university students living in residence. J.Can.Diet.Assoc., v.38, p.47-54, 1977.

09. HERNON, J.F. et al Nutrient intakes and foods selected by college students: comparisons smong subgroups divides by energy intake. J.Am.Diet.Assoc. v.86, n.2, p.217-21, 1986.

10. HERTZLER, A.A.; FRARY, R. Dietary status and eating out practices of college students. JAm.Diet.Assoc., v.92, p.867-9, 1992.

11. ISHIGAKI, S.; SUZUKI, J. A study on nutrient intake conditions and caloric consumption of girl students. Jap.J.Nutr., v.33, n.2, p. 79-83, 1975. 
12. JAKOBOVITS, C. et al Eating habits and nutrient intakes of college women over a thirty-year period. J.Am.Diet.Assoc.. v.71, p.405-11, 1987.

13. KRAUSE, M.V.; MAHAN, K. Alimentos, nutrição e dietoterapia: um livro texto do cuidado nutricional. 7.ed. Sáo Paulo, Roca, 1991.

14. MAEKAWA, M. et al Anutritional ourvey and the dailey schedule of college giris. Jap.J.Nutr. v.35, n.6, p. 307-14, 1977.

15. MILLER, T.M. et al Survey on body image, weight and diet of college students. JAm.Diet.Assoc, n.77, p.561-69, 1980.

16. MTCHELL, H.S. et al Nutriçào. 16.ed. Rio de Janeiro, Interamericana, 1978.

17. NAKAMAE, D.D. Perfil do estudante de enfermagem. Sào Paulo, 1976. Dissertaçäo(Meotrado) - Escola de Enfermagem, Universidade de São Paulo.

18. NÚCLEO DE APOIO AOS ESTUDOS DE GRADUAÇĀO (NAEG), Universidade de São Paulo (USP). Ingressantes - 1991 (Projeto Tempo Zero-Segunda Fase). Caderno 1 - Reoultados Globais para a USP. Säo Paulo, 1991.

19. ORGANIZACION MUNDIAL DE LA SALUD Problemas de salud de la adolescencia. Ginebra. 1965. 30p. (Seriede Informes Tecnicos, 308)

20. OSTRON, S.; LABUZA, T.P. Analysis of a seven-day diet survey of college students. Food Technol, v.31, n.5, p. 68.76, 1977.

21. SIQUEIRA, A.A.F.; TANAKA, A.C.d'A. Mortalidade na adolescéncia com especial referéncia à mortalidade materna, Brasil, 1980. Rev.Saúde Públ. v.20, n.4, p. 274-9, 1986.

22. WEST, K.M. Computing and expressing degree of fatness (letter to the editor). J.A.M.A. v.243, p.1421, 1980. 